SASKATCHEWAN NATURAL

HISTORY SOCIETY

P.O. BOX 1321, REGINA, SASKATCHEWAN, S4P 3B8

\title{
BOARD OF DIRECTORS
}

\section{ICERS}

orary President

ident

President

Vice-President

and Vice-President

surer

-esponding Secretary

prding Secretary

\section{OINTED DIRECTORS}

Jay Editor

Jay Brokshop

slation

servation

angered Specie's

slands Park

al Socieries

\section{ahership}

sletter Editors

ial Publications
Elizabeth Cruickshank

Gary W. Seib

Margaret Belcher

Ole Nielsen

Frank Switzer

George R. Dodd

Gary Wobeser

Dale Hjertaas

J. Bernard Gollop

Frank Brazier

Lorne Scott

Saskatchewan Museum of Natural History, Regina Frank Switzer

1301 Shannon Rd., Regina, S4S 5K9 James R. Jowsey 2635 Nineteenth Ave., Regina, S4T I X2 George F. Ledingham 2335 Athol St., Regina, S4T 3G4 Lorne Scott

Saskatchewan Museuni of Natural History, Regina Lynn Oliphant

330 Saskatchewan Cres. W. Saskatoon, S7M 0A4

\section{Lloyd O. T. Peterson}

W.m. and Joyce Anaka

\section{RESENTATIVES AT LARGE}

E. Andrews

rge Ferguson

843 Athabasca St. W., Moose Jaw, Sask. S6H 2E2

ig Francis

73 Cardinal Cresc., Regina, Sask. S4S 4 Y6

Hamilton

Broadview. Sask. SOG OKO

ne Harris

Nokomis, Sask. S0G 3R0

Hayward

Box 93, Raymore, Sask. S0A 3J0

tin McNicholl .... Dept. of Zoology, Univ. of Alberta, Edmonton, Alta. T6G 2El

istine Pike

Box 117, Waseca, Sask. SOM 3A0

Riome

Box 2103 , Nipawin, Sask. SOE 1 E0

Rump ................... 3320 Angus St., Regina, Sask. S4S IP8

heer Sealy ...... Dept. of Zoology, Univ. of Manitoba, Winnipeg, Man. R3T 2O2

y Wobeser ................... 209 Tucker Cresc., Saskatoon, Sask. S7H 3J1

\section{SIDENTS OF LOCAL SOCIETIES}

Qu'Appelle

an Head

ple Creek

se Jaw

ina

atoon

it Curren

$\mathrm{kton}$

versity Biology Clubs
Bernard de Vries

Mary Skinner

D. Bromley

Patricia Kern

W. Lloyd Hipperson

Don McRobbie

Jan Looman

Phil Pawluck

Richard Collins

Dale Botting
Box 1043, SOG 1 SO

P.O. Box 777. SOG $2 \mathrm{KO}$

SON INO

1053 Chestnut Ave., S6H 1 A7

3708 Albert St., S4S 3P9

525 - 4th Ave. N., S7K 2 M5

491 - 2nd Ave. S.E., S9H 3J7

163 Peaker Ave., S3N 1 S6

University of Regina, S4S OA2

University of Saskatchewan, S7N OW0 